\title{
Effects of season and follicle size on the metabolomic profile of porcine follicular fluid
}

\author{
M.J. Bertoldo ${ }^{1,2}$, L. Nadal-Desbarats ${ }^{3}$, N. Gérard ${ }^{1}$, A. Dubois ${ }^{3}$, P.K. Holyoake ${ }^{2}$ and \\ C.G. Grupen ${ }^{2}$ \\ ${ }^{1}$ INRA, UMR85 Physiology of Reproduction and Behaviour, 37380 Nouzilly, France; ${ }^{2}$ Faculty of Veterinary \\ Science, The University of Sydney, Camden, NSW 2570, Australia; INSERM U930, PPF Analysis of \\ Biological Systems, Faculty of Medicine, 37044 Tours, France
}

The domestic sow exhibits a decline in reproductive performance during the late summer and early autumn months, which in production systems is manifested as a decrease in farrowing rates. A recent study found that impaired oocyte quality may contribute to the seasonal infertility phenomenon, as oocytes collected in summer displayed a reduced capacity to form blastocysts in vitro compared with oocytes collected in winter (Bertoldo et al. 2010). The effect of season on oocyte quality was apparent in oocytes from large antral follicles (Bertoldo et al. 2010), which normally have superior developmental potential compared with oocytes from small antral follicles (Bagg et al. 2007). During the latter stages of folliculogenesis the cumulus oocyte complex is bathed in follicular fluid (FF), which provides a specialised and dynamic microenvironment that supports the final stages of oocyte maturation. While FF is known to consist of serum exudates and locally produced factors secreted by the follicular cells, detailed characterization of the composition of FF may provide useful insights into follicle growth and differentiation and the acquisition of oocyte developmental competence.

Metabolomics is the non-targeted identification and quantification of all the metabolites present in a biological sample. In contrast to transcriptomic or proteomic analyses, metabolomic analysis has the advantage of identifying the biological endpoints that occur as a result of environmental change or altered gene expression (Baskind et al. 2011). High resolution proton nuclear magnetic resonance ('H-NMR) spectroscopy provides a unique tool for studying the composition of biofluids as it is capable of identifying and quantifying all the metabolites present in an untreated sample. To date there are relatively few reports of the use of this technology to analyse FF samples (Baskind et al. 2011). Therefore, using the previously observed seasonal and follicle size effects on oocyte quality to classify models of good and poor follicular environments, the aim of the present study was to characterize the metabolomic profiles of porcine FF samples collected from small and large follicles in summer and winter by ${ }^{1} \mathrm{H}-\mathrm{NMR}$ spectroscopy.

The FF samples were obtained from adult Large White/Landrace cross-bred sows as described previously (Bertoldo et al. 2011). The ovaries of sows culled for non-reproductive reasons (eg. lameness) were collected at slaughter, 4 days after weaning, in summer and winter (on at least three separate occasions in each season). The contents of small $(3-4 \mathrm{~mm})$ and large $(5-8 \mathrm{~mm})$ diameter follicles were aspirated and pooled separately (small FF and large FF) for each ovary pair. Cellular material was removed by centrifugation and the FF samples were stored at $-20^{\circ} \mathrm{C}$. Only FF samples of sufficient volume $(500 \mu l)$ were analysed (summer-small $(n=8)$, summer-large $(n=15)$, winter-small $(n=9)$ and winter-large $(n=14))$. For ${ }^{1} H-N M R$ spectroscopy, the FF samples were thawed at room temperature, centrifuged at $3000 \mathrm{~g}$ for $5 \mathrm{~min}$, and prepared by mixing $500 \mu \mathrm{l}$ of FF, $100 \mu \mathrm{L}$ of $\mathrm{D}_{2} \mathrm{O}$ solution and $100 \mu \mathrm{L}$ of phosphate buffer to obtain a pH value of $7.4 \pm 0.5$. The spectra were acquired using a Bruker DRX-500 spectrometer (Bruker SADIS, Wissembourg, France) operating at 11.7 T, with a Broad Band Inverse probe head equipped with a Z gradient coil, and recorded with $90^{\circ}$ pulse $(\mathrm{p} 1=10 \mu \mathrm{s}, \mathrm{pl}=0 \mathrm{~dB})$ using a pulse-and-acquire sequence with residual water pre-saturation. Spectra were collected with 128 transients (and 8 dummy scans) in $32 \mathrm{~K}$ data points with a spectral width of $7500 \mathrm{~Hz}$, and a recycling time of $15 \mathrm{~s}$, and processed using WinNMR version 3.5 software (Bruker Daltonik, Karlsruhe, Germany). All spectra were corrected for phase distortion and the baseline was manually corrected for each spectrum. Spectral peaks were assigned according to the literature values of chemical shifts in various media and biofluids and quantified by using the electronic reference to 
access in vivo concentrations signal. A linear mixed model was fitted to the data using the statistical software package GenStat 14th edition (VSN International, Ltd., Hemel Hempstead, UK). For each metabolite, the treatment effects "follicle size" and "season" were assessed for significance $(\mathrm{P}<0.05)$, as was treatment interaction. The term "pig", which specified individual animals, was included as a random term. When a treatment interaction was detected, approximate LSDs were used to compare pairs of treatment means.

Preliminary results show that follicle size affected the concentrations of eleven metabolites, independent of season. Glucose was the only metabolite affected by follicle size alone that had a greater concentration in large FF compared with small FF $(0.884 \pm 0.040$ vs $0.762 \pm 0.050 \mathrm{mM}$; $\mathrm{P}<0.05)$. In contrast, the concentrations of acetate, hypoxanthine, inositol, lactate, trimethylamine and the amino acids alanine, leucine, lysine, methionine and phenylalanine, were all lower in large FF than in small FF. The percentage difference in metabolite concentrations between small FF and large FF ranged from $14 \%$, for glucose, to $78 \%$, for hypoxanthine. Succinate was the only metabolite affected by season, independent of follicle size. The concentration of succinate in FF collected in winter was $16 \%$ greater than that in FF collected in summer $(0.253 \pm 0.015$ vs 0.218 $\pm 0.007 \mathrm{mM} ; \mathrm{P}<0.05)$. Significant interactions between follicle size and season were found for the concentrations of glutamate, glycine, uridine and $\mathrm{N}$-acetyl groups. The concentration of glutamate was greater in small FF compared with large FF, and the difference was greater in summer $(\mathrm{P}<0.05)$. The concentrations of glycine and uridine in small FF collected in summer were greater than those in small FF collected in winter, and were also greater than those in large FF collected in either season $(\mathrm{P}<0.05)$. Likewise, the concentration of $\mathrm{N}$-acetyl groups in small FF collected in summer was greater than that in small FF collected in winter $(\mathrm{P}<0.05)$, but the concentrations in large FF collected in either season were intermediate.

These findings extend those of previous studies in other species that show the concentrations of numerous metabolites in FF change as follicle development progresses. The increase in glucose level and the concomitant decrease in lactate level indicate that there is a distinct shift in carbohydrate metabolism and/or uptake by the granulosa cells as follicle size increases. Many of the other metabolites affected by follicle size alone, including acetate, alanine, leucine, lysine, methionine and phenylalanine, are precursors of citric acid cycle intermediates, suggesting that the activity of this pathway increases during the final stages of folliculogenesis. In addition, the change in the concentration of hypoxanthine, a non-specific inhibitor of phosphodiesterase activity, is consistent with a follicular environment that is more permissive of oocyte meiotic resumption.

The seasonal differences in the FF metabolomic profiles differed markedly from those associated with follicle size alone. The change in the level of succinate, an intermediate of the citric acid cycle, suggests that energy generation via this pathway is sub-optimal during the summer months. As the developmental potential of oocytes from large follicles is reduced during the summer months (Bertoldo et al. 2010), the seasonal changes in the metabolites of FF may also be associated with sub-optimal oocyte maturation conditions. We propose these metabolite changes are suggestive of a follicular environment that is less able to protect cells from oxidative stress. While further studies are needed to clarify the impact of the observed metabolite changes on oocyte quality, the findings demonstrate the power of ${ }^{1} \mathrm{H}-\mathrm{NMR}$ spectroscopy to expand our understanding of the complex and dynamic microenvironment of the developing follicle.

\section{References}

Bagg MA, Nottle MB, Armstrong DT \& Grupen CG 2007 Relationship between follicle size and oocyte developmental competence in prepubertal and adult pigs. Reproduction Fertility and Development 19 797-803.

Baskind NE, Mcrae C, Sharma V \& Fisher J 2011 Understanding subfertility at a molecular level in the female through the application of nuclear magnetic resonance (NMR) spectroscopy. Human Reproduction
Update 17 228-241.

Bertoldo M, Holyoake PK, Evans G \& Grupen CG 2010 Oocyte developmental competence is reduced in sows during the seasonal infertility period. Reproduction Fertility and Development 22 1222-1229.

Bertoldo M, Holyoake PK, Evans G \& Grupen CG 2011 Follicular progesterone levels decrease during the period of seasonal infertility in sows. Reproduction in Domestic Animals 46 489-494. 